\title{
Radioprotection : quarante ans de publication
}

J'avais conclu mon travail de responsable du club « histoire » de la SFRP par la publication d'un article : «Un peu d'histoire : la revue Radioprotection, 1966 à 1999 », dans le numéro 2 de 2005, pp. 151-171. Protégé par ces vingt pages, je me croyais à l'abri. Lourde était mon erreur : lors de la récente réunion du comité de rédaction, Henri Métivier, après m'avoir félicité pour avoir adhéré à l'association dès sa création, et pour avoir participé au premier comité de rédaction, a affirmé que cela me rendait totalement prioritaire pour rédiger un second article sur la revue, évidemment différent du premier, par exemple plus anecdotique (Fig. 1).

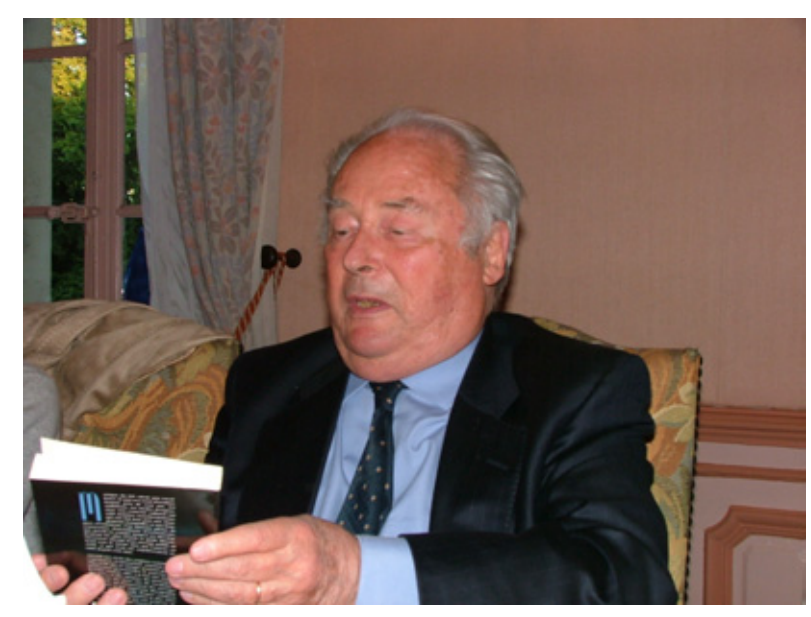

Figure 1 - Daniel Blanc, 40 ans de Radioprotection.

Il m'était difficile de résister. Je me suis donc retrouvé devant une page blanche, à noircir, comme les suivantes, et j'ai repris le chemin de l'historiographie chronologique...

L'évolution de la revue a été fortement marquée par la personnalité de chaque président de la Commission des publications, devenue plus récemment le Comité de rédaction. Leur nombre est de cinq; je découperai donc mon propos en cinq parties. Le président est secondé par un exécutant, sorte de secrétaire de rédaction, dont dépend le déroulement harmonieux des opérations, jusqu'à la sortie de chaque numéro. Dans le titre de chaque morceau, son nom sera placé entre parenthèses, après celui du président.

DOI: 10.1051/radiopro:2006011

RADIOPROTECTION - VOL. $41-\mathrm{N}^{\circ} 3(2006)$ 


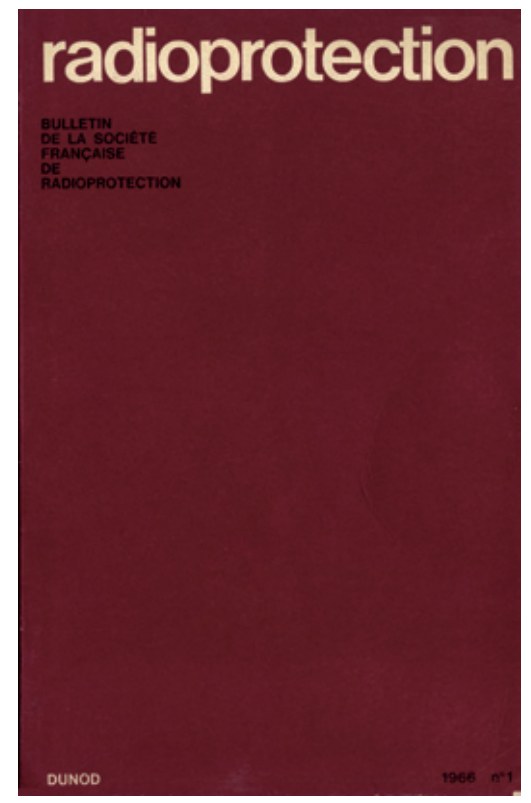

Figure 2 - La couverture du premier numéro.

\section{1966-1979 : Henri François (Norbert Chassende-Baroz, puis Anne-Marie Beau)}

La couverture de la revue (Fig. 2), austère, rappelle la robe de bure d'un trappiste. La commission des publications, mise en place par le conseil, compte huit personnes, qui mirent à leur tête Henri François, docteur es sciences, bourguignon, et chef d'une section de dosimétrie au CEA à Fontenay-aux-Roses. Voici leur liste :

Henri François (CEA)

Maurice Avarguès (CEA, université)

Daniel Blanc (université)

Pierre Bonet-Maury (CNRS)
Norbert Chassende-Baroz (armées)

Henri Joffre (CEA)

Michel Macqueron (CEA)

Jules Manquené (CEA)

Les représentants du CEA sont en majorité, mais cela n'a généré aucun problème, Henri François étant d'esprit très universitaire.

Norbert Chassende-Baroz était pharmacien général. La réalisation matérielle de la revue le passionnait, et il a effectué son travail de secrétaire de rédaction avec une aménité et une efficacité tout à fait remarquables. Il souffrait d'un asthme grave et il arrivait à nos réunions muni d'une bouteille d'oxygène, indispensable en cas de crise. La SFRP lui doit beaucoup. 
Pierre Bonet-Maury avait été le second président de la SFRP, après le général Chassende-Baroz qui avait donné l'impulsion initiale. Pharmacien chevronné, il était propriétaire d'une pharmacie dans Paris ; maître de recherche au CNRS, il était chargé de la radioprotection du campus d'Orsay. Son violon d'Ingres était le judo ; il a écrit un «Que sais-je ? » sur ce sport, qui a été un succès et a connu plusieurs rééditions.

Maurice Avarguès, biologiste très connu, professeur d'université, était détaché au CEA. Il était très attentif au niveau scientifique de notre revue. Henri Joffre et votre serviteur nagions dans les questions de mesure, et plus particulièrement de dosimétrie. Michel Macqueron, ancien officier de marine, adjoint de Jacques Pradel, secondait Norbert Chassende-Baroz; il nous apportait son calme, et ses connaissances techniques. Jules Manquené représentait les médecins, il dirigeait alors le service de médecine du travail du centre de Fontenay-aux-Roses ; il devait devenir le responsable de l'ensemble de la médecine du travail au CEA.

Cette première commission ne comptait pas de représentants d'EDF et des entreprises. Cette lacune fut comblée lorsque la revue démontra son intérêt pour les spécialités de ces domaines.

En 1966, on se trouvait en pleine époque Gaullienne, durant laquelle le nucléaire bénéficia de moyens matériels considérables; cet état d'euphorie conduisait certains radioprotectionnistes à faire du triomphalisme. La figure 3 reproduit le sommaire du numéro 1. Le premier article, par Francis Duhamel, ancien chef du SCRGR, "point de vue sur la prévention des accidents d'origine nucléaire » en est le reflet.

Pour éviter les prélèvements extérieurs, la commission s'est efforcée de recruter des annonceurs par l'action directe de ses membres. On ne s'improvise pas démarcheur, et le résultat fut catastrophique. Il fallut donc se résigner à accepter les pourcentages prélevés par les agences de publicité...

Pour alléger les tâches de la commission des publications, aux objectifs plus larges, le conseil de la SFRP créa en 1969 un comité de rédaction chargé de traiter les problèmes au jour le jour (N. Chassende-Baroz et M. Macqueron, épaulés par R. Hantz, collaborateur du Dr J. Manquené). Cela a permis un fonctionnement matériel harmonieux, malgré le comportement parfois erratique de l'éditeur. Je vous renvoie à mon article « sérieux » de 2005.

Henri François faisait preuve d'audace ; il allait chercher des articles à droite et à gauche, et même à l'étranger, ce qui le conduisit à publier des articles en anglais... contre la volonté du conseil de la SFRP, qui, dans sa grande majorité, se déclarait pour la défense de la langue française. Henri François agissait par impulsions, sans prévenir, et (ou) sans autorisation, ce qui déplaisait aussi beaucoup. À partir de 1976, un nombre croissant de conseillers estimaient qu'il 


\section{RADIOPROTECTION}

BULLETIN DE LA SOCIETTE FRANÇAISE

DE RADIOPROTECTION

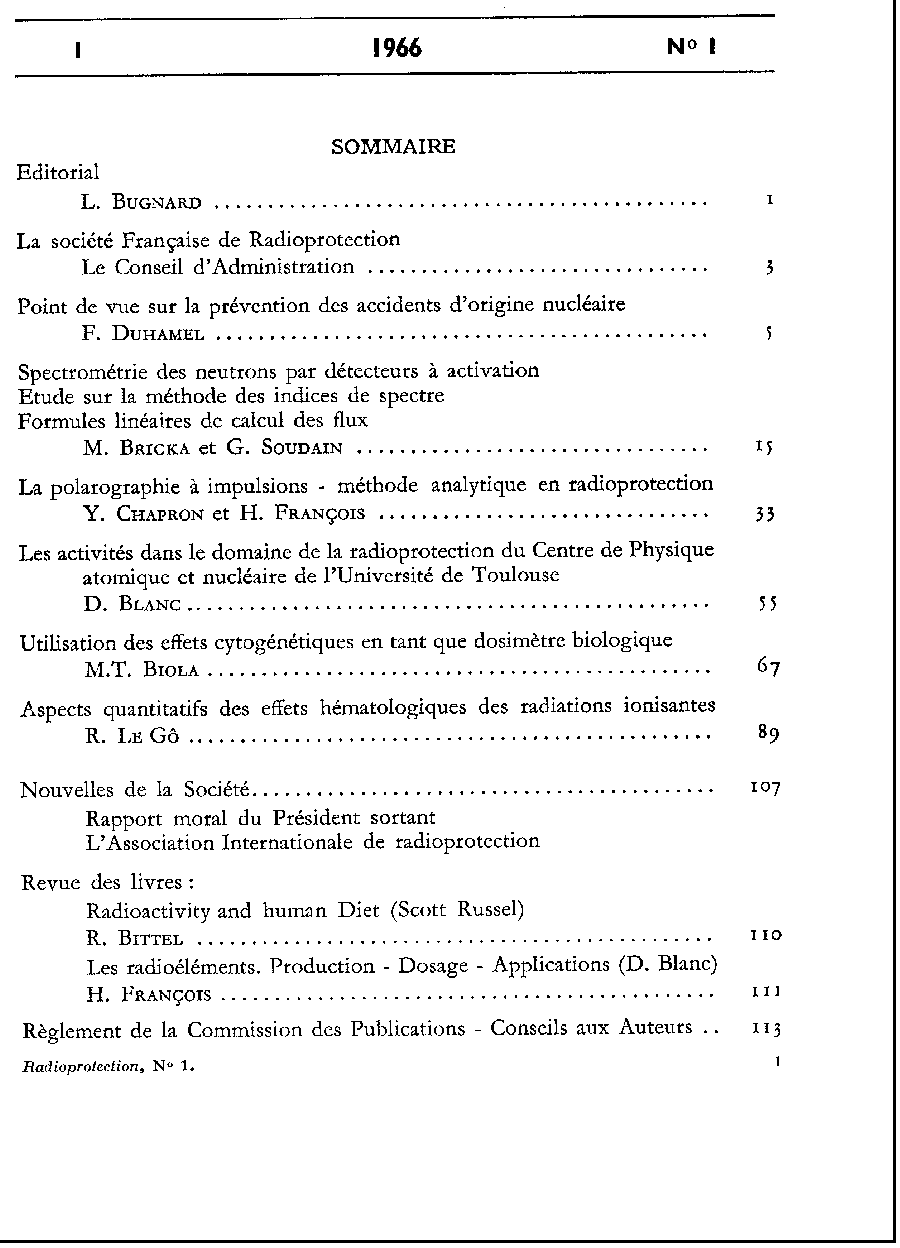

Figure 3 - Sommaire du premier numéro. 
fallait limiter dans le temps les fonctions de responsable de la revue. Henri François, mis en minorité en 1979, dût céder la place...

\section{1979-1982 : André Teste du Bailler (Anne-Marie Beau)}

Le nouveau responsable, ingénieur à l'EDF, est membre du Conseil d'administration; il s'emploie à supprimer tout ce qui peut déplaire. À ce point de vue, c'est une période calme. Madame Anne-Marie Beau, documentaliste au CEA, était venue, deux ans plus tôt, se joindre à l'équipe pour épauler Norbert Chassende-Baroz, puis le remplacer lorsqu'il fut trop handicapé physiquement ; il devait décéder en 1982. Madame Beau a conservé ces fonctions durant plus de vingt ans : la régularité de parution de la revue, sa bonne présentation, sont dues pour beaucoup à son attention et à son sens des réalités.

Les ennuis viennent de l'éditeur, trop préoccupé par ses intérêts matériels ; la situation est souvent tendue, et difficilement vivable. On divorce donc, et, sur le conseil de Jacques Pradel, la SFRP choisit le GEDIM, groupement d'édition qui dépend de l'association des anciens élèves de l'École des mines de Saint-Étienne, dont Jacques Pradel est membre. Le choix s'avère excellent, l'ambiance devenant beaucoup moins mercantile.

Le responsable est en harmonie avec le conseil (voir plus haut) et en devient président en 1982. Il quitte alors son poste de responsable de la revue. Pierre Bovard, ingénieur au CEA, lui succède.

\section{1982-1987 : Pierre Bovard (Anne-Marie Beau)}

Le $1^{\text {er }}$ janvier 1982, le GEDIM propose une nouvelle couverture (vert et jaune), plus aimable (Fig. 3). Les membres de la commission de la revue continuent à assurer l'expertise scientifique des manuscrits, et cette commission grossit progressivement, par l'addition d'experts ouvrant de nouveaux domaines. La réunir devient une opération lourde ; la nourrir, encore plus...

À la fin de 1984, Pierre Bovard décide d'officialiser un comité de rédaction comptant, à ses côtés, Mme Beau, Mme Odile Pennaneac'h, également du CEA, ainsi que Jacques Lochard, nouveau venu dans la commission des publications.

En 1986, Pierre Bovard, retraité du CEA, devient un élu local. Il souhaite se consacrer entièrement à cette tâche municipale.

\section{1987-1996 : Daniel Blanc (Anne-Marie Beau, jusqu'à la fin de 1995)}

Il est bien délicat de parler de soi. D'ailleurs, tout ce que j'ai fait était parfait. Alors, pourquoi en parler? 
La Commission des publications continue à grossir ; en 1987, elle compte 24 membres français et 17 membres étrangers. Le Conseil d'administration la supprime, et ne garde que le comité de rédaction (4 membres). Ce comité va, lui aussi, grossir, et pour des raisons analogues, mais beaucoup plus lentement. Il compte aujourd'hui 14 membres.

Pour des raisons financières, le GEDIM arrête ses activités le $1^{\mathrm{er}}$ septembre 1991 ; c'est, hélas, le sort de bien des petites maisons d'édition, pas trop pirates. Le successeur fut la société «Les éditions de physique » (qui changea de nom en 1998 et devint EDP Sciences ${ }^{1}$ ). Le choix était excellent ; nous n'avons qu'à nous féliciter de l'état d'esprit de nos interlocutrices, et de leur très grande compétence. Je peux parler de Florence Grosseaux, puis d'Agnès Henri et de Sophie Hosotte.

En 1993, Henri Métivier devint vice-président du Comité de rédaction, avec droit de succession. Par contre, Madame Beau ayant pris sa retraite, quitte ses fonctions à la fin de 1995. Son travail fut ensuite réparti entre les éditrices d'EDP Sciences et Henri Métivier.

Pendant cette période nous avons changé de couverture une nouvelle fois, ce fut dur puisqu'il nous a fallu 3 ans pour en arriver au projet que vous avez reçu pour la dernière fois lors du précédent numéro (Fig. 4).

En 1996, j’ai estimé qu'il était temps de prendre un peu de recul en cédant ma place de président à mon vice-président ce qui nous amène au dernier chapitre.

\section{Depuis octobre 1996 : Henri Métivier (et divers)}

Nous voici parvenus à l'époque contemporaine, trop récente pour permettre d'effectuer un travail historique. Ce comité, je l'ai dit plus haut s'est ouvert à toutes les composantes de la radioprotection française, université, CEA, IRSN, EDF, AREVA, Armées. Grâce à notre secrétaire général, Jacques Lombard, nous avons notre traditionnelle photo indoor ou outdoor (c'est selon), nos emplois du temps ne nous permettent pas d'être toujours au complet (Fig. 5)

En guise de conclusion, je voudrais évoquer les « délocalisations » du Comité de rédaction, c'est-à-dire ses réunions, annuelles, au sein d'un haut lieu scientifique et (ou) technique féru de radioprotection.

Voici la liste des sorties :

- 1994 : centrale électronucléaire de Golfech, la place d'ALARA,

- 1995 : université de Limoges, laboratoire de dosimétrie (Prs Decossas et Vareille),

\footnotetext{
1 EDP Sciences est le sigle de Édition Diffusion Presse Sciences, la propriété de l'entreprise est partagée entre la Société française de physique, la Société française de chimie et la Société de mathématiques appliquées et industrielles. 

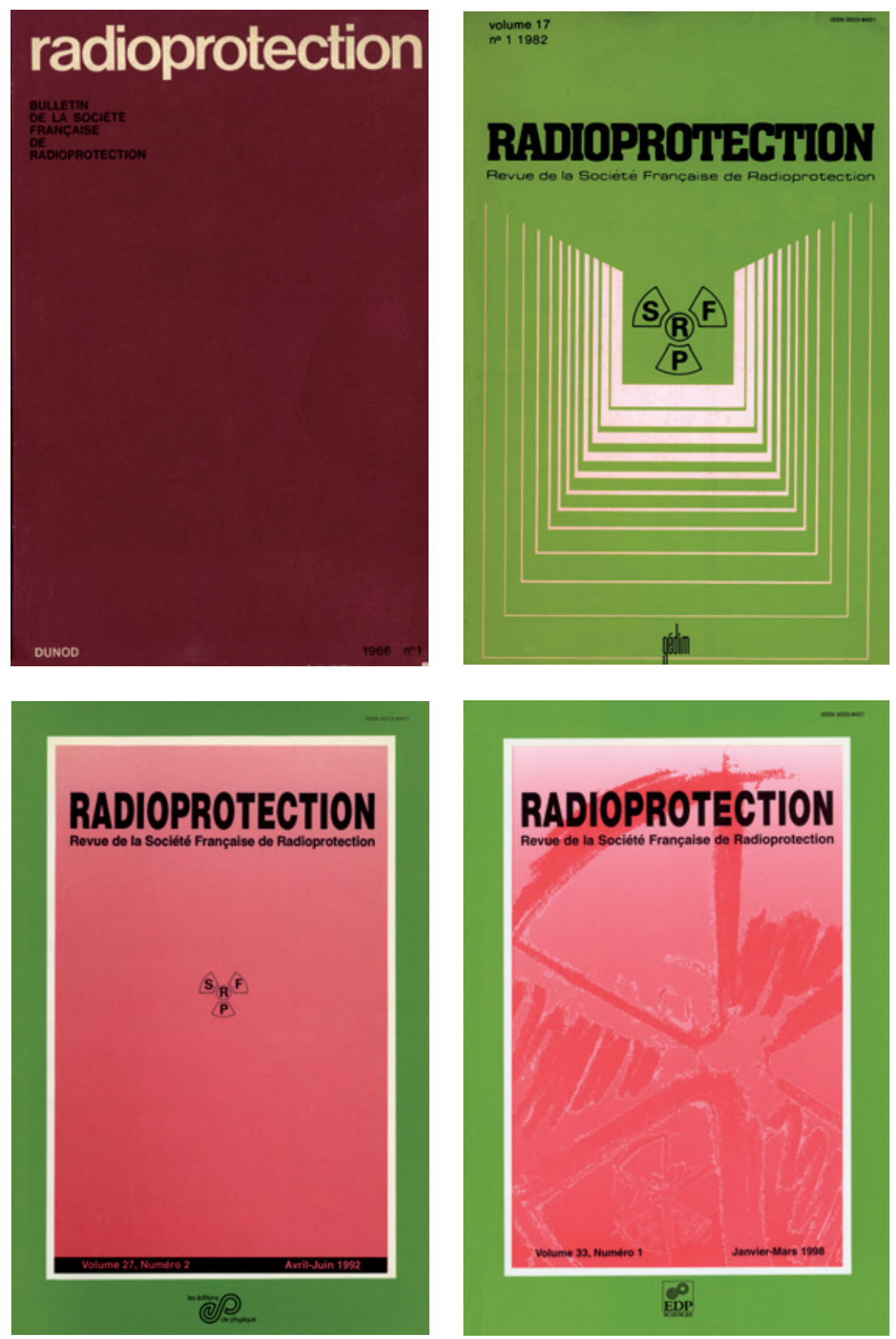

Figure 4 - Couvertures successives. La nouvelle couverture se trouve au début du numéro que vous lisez. 
D. BLANC
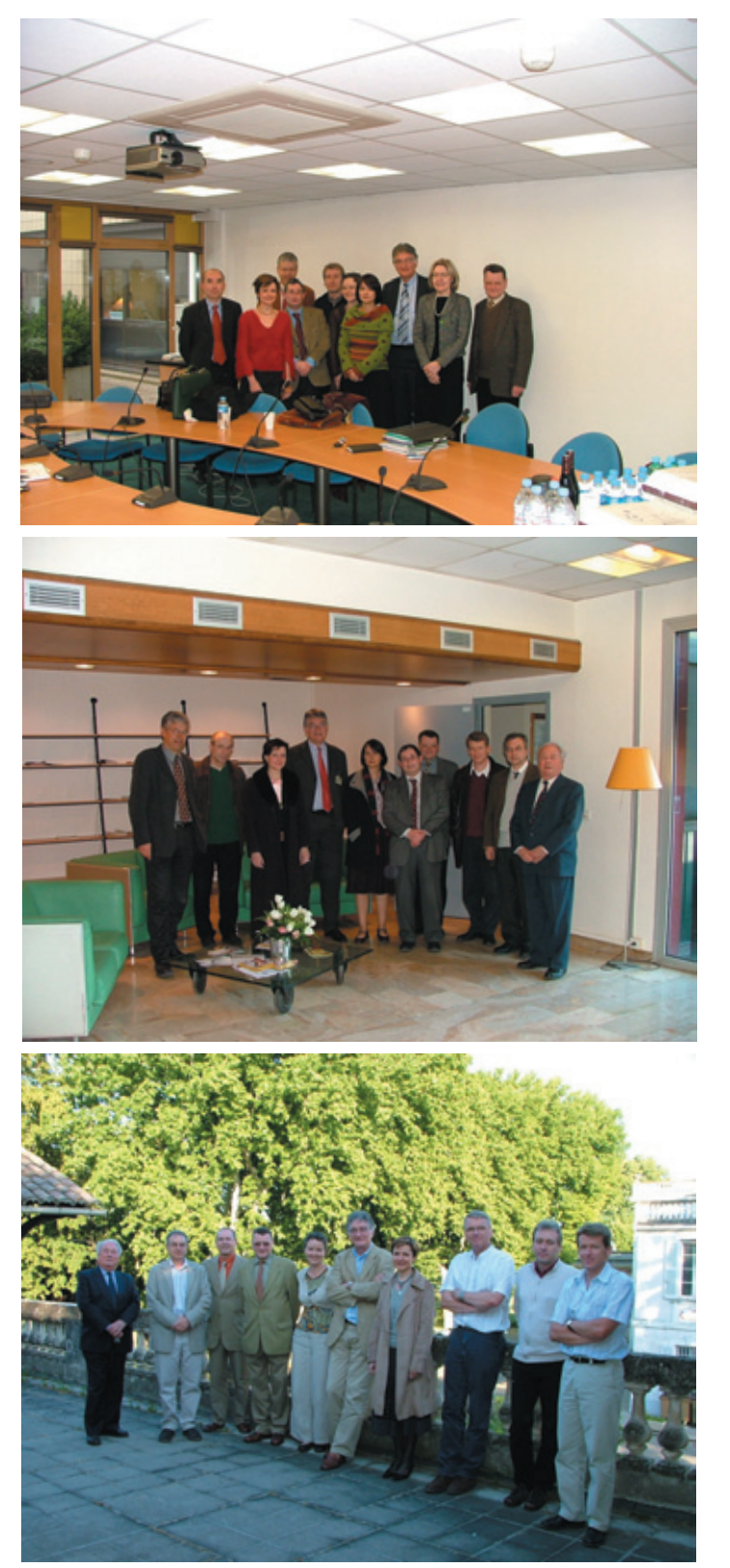

Figure 5 - Indoor ou outdoor, un Comité toujours studieux. 
- 1996 : université et port de Brest, visite d'un sous-marin nucléaire et du De Gaulle en construction, tout cela en une après-midi (Georges Tymen),

- 1997 : Comurhex, Malvési organisée par le regretté Benoît Gibert,

- 1999 : Cadarache, visite détaillée de la maison d'hôtes, le centre étant en grève,

- 2000: Brennilis (monts d'Arrée) en plein démantèlement, encore une organisation Georges Tymen,

- 2001 : Melox sous la houlette de Patrick Raynaud,

- 2002 : l'hôpital Percy, dont l'espace des grands brûlés grâce à Patrick Gérasimo,

- 2003 : le comité radioprotection d'EDF, délocalisation en plein Paris, Jeannine Lallemand,

- 2004 : Le Vésinet nouveau (IRSN), sous la conduite de Marie-Christine Robé,

- 2005 : à Cadarache, les installations Amande (IRSN) et Leca (CEA) grâce à Henri Maubert,

- 2006: Pierrelatte, présentation du laboratoire de radiotoxicologie expérimentale de l'IRSN (François Paquet) et de l'usine Eurodif (Areva) grâce à Jean-Jacques Radecki.

C'est vous dire qu'ainsi, les membres du comité de rédaction sont devenus des puits de science.

Enfin, autre évolution de notre revue, sa mise en place sur Internet, nous savons maintenant mieux ce que souhaite notre lectorat. Le classement des articles les plus téléchargés va nous pousser à délivrer des médailles...

Une bonne conclusion, n'est-il pas vrai?

Daniel Blanc

SFRP 